\title{
Irrigated DiamondTemp catheter and return to ablation under temperature control. First Polish experience with DiamondTemp catheter in pulmonary vein isolation
}

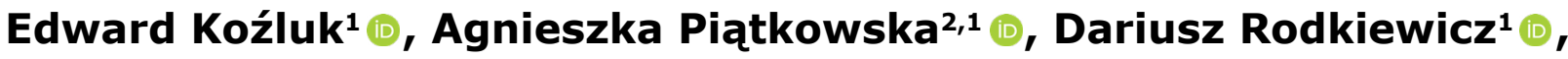 \\ Grzegorz Opolski ${ }^{1}$ (i)
}

${ }^{1}$ I Chair and Department of Cardiology, Medical University of Warsaw, Warsaw, Poland

${ }^{2}$ Department and Clinic of Emergency Medicine, Wrocław Medical University, Wrocław, Poland

\begin{abstract}
We present the first Polish experience with ablation performed using DiamondTemp catheter. The study was conducted with 3 male patients diagnosed with atrial fibrillation (AF). In the first 2 patients typical transseptal punctures were performed, followed by mapping with the Advisor catheter and EnSite-Precision system. One patient had a residual atrial septal leak, therefore ablation without fluoroscopy was attempted. High-power, short-duration ablation under temperature control was performed around pulmonary vein (PV) ostia. The power was $49-53 \mathrm{~W}$, the temperature was $45-48^{\circ} \mathrm{C}$. Duration of procedures/fluoroscopy were: $146 / 8.9,177 / 5.9$, $132 / 0.0 \mathrm{~min}$. In the reference group, 10 recent AF identical ablation procedures performed with traditional equipment resulted in 143.0 $\pm 27.0 / 6.0 \pm 4.4 \mathrm{~min}$. Duration of DiamondTemp applications were 14.7, 32.7, 30.8 min (reference group $37.3 \pm 11.4 \mathrm{~min}$ ). Procedural endpoints were achieved in all but one patient with incomplete isolation of the low segment of the right inferior PV. There were no procedural complications noted. In conclusion, the DiamondTemp saline-irrigated catheter is safe and effective for high-power short-duration ablation in patients with AF. Furthermore, this technology makes it possible to complete the procedure without fluoroscopy. However these findings must be confirmed in larger group of patients.
\end{abstract}

Keywords: atrial fibrillation $\cdot$ zero fluoroscopy $\cdot$ pulmonary vein isolation $\cdot$ high-power short-duration ablation - temperature control RF ablation

\section{Citation}

Irrigated DiamondTemp catheter and return to ablation under temperature control. First Polish experience with DiamondTemp catheter in pulmonary vein isolation. Eur J Transl Clin Med. 2021;4(2):44-51.

DOI: $10.31373 /$ ejtcm/136234 


\section{Introduction}

In the recent years there has been a technological breakthrough in the field of atrial fibrillation (AF) ablation due to the introduction of the ablation with high-power and short-duration [1-9]. In classical ablation, thermal injury occurs in two phases: resistive and conductive, with the second one being the dominant mechanism. In the resistive phase, RF current delivery leads to immediate heating of the superficial tissue layers approximately $1.0-2.0 \mathrm{~mm}$ depth. This phase creates a heat source that then extends passively to deeper layers as conductive heating [1-2]. Conductive heating injury is time dependent. Irreversible myocardial injury occurs at temperature $\geq 50^{\circ} \mathrm{C}$ [10]. At lower temperature the tissue injury often is reversible. When the power is increased the duration of current application is reduced and the resistive heating dominates the lesion creation [12]. Thus, the depth of permanent injury is more predictable, and the risk of collateral tissue damage is reduced. High-power ablations are classified as those performed with a power of $\geq 50 \mathrm{~W}$ [1-9].

Since temperature control during ablation is a better marker of effectiveness and safety in regard to tissue damage, it was favored overpower control for as long as until the end of the last century [1115]. The gradual introduction of irrigated catheters that followed, resulted in loss of the possibility to accurately measure temperature at the electrode-tissue control interface, due to technological difficulties [15]. This impasse seems to be now solved by introduction diamond electrodes placed at the tip of catheter, which allows for efficient and fast heat removal [8-9].

In our previous article we presented a different catheter featuring very high-power and short-duration ablation technology [16]. This ablation method increases the proportion of resistive phase versus conductive phase of tissue damage, making RF application more predictable and safer. An additional benefit is the shorter application and treatment time.

In this technical report we aim to present a different solution: DiamondTemp Catheter (Medtronic) (Fig.1-3).

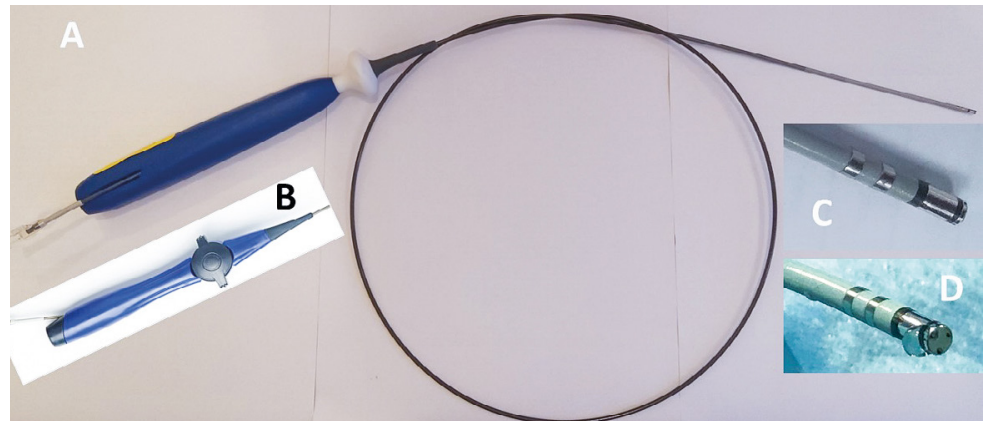

Figure 1. A - unidirectional version of the DiamondTemp catheter. B - bidirectional version of the catheter handle. $\mathrm{C}$ - close-up of the distal part of the DiamondTemp catheter. As shown, the ablation catheter tip consists of two electrodes: 0.6 and 3.0 $\mathrm{mm}$ with a $0.5 \mathrm{~mm}$ diamond in between. Proximal to the second electrode there is another diamond ring. Next, there are two diagnostic electrode rings. $D-$ front of the catheter tip with its three thermocouple

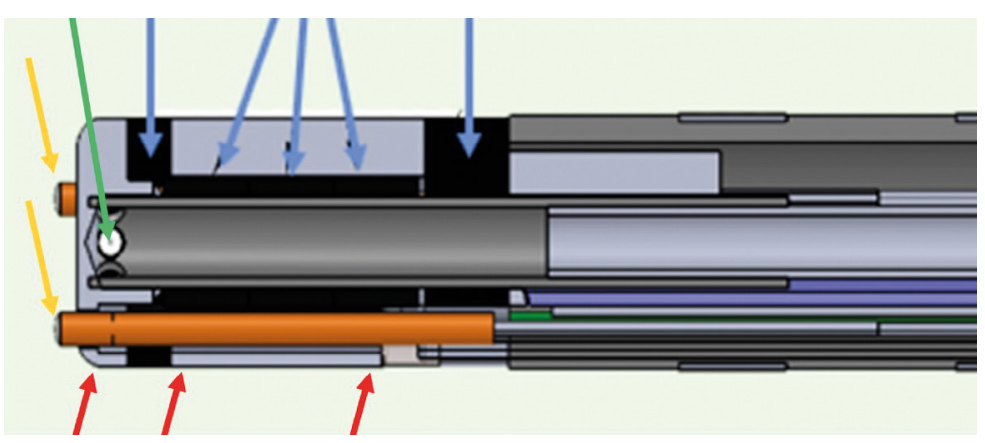

Figure 2. Longitudinal section of the DiamondTemp catheter. Yellow arrows indicate termocouples in front of the distal electrode. Red arrows - the RF platinum-iridium rings. The left one indicates the distal $0.6 \mathrm{~mm}$ electrode, the next two indicate the beginning and the end of the proximal RF electrode. Green arrow indicates one of its six irrigation ports. Blue arrows indicate positioning of the diamond. The left one indicates the distal diamond, the next three indicate the central diamond, the right one indicates the proximal diamond.

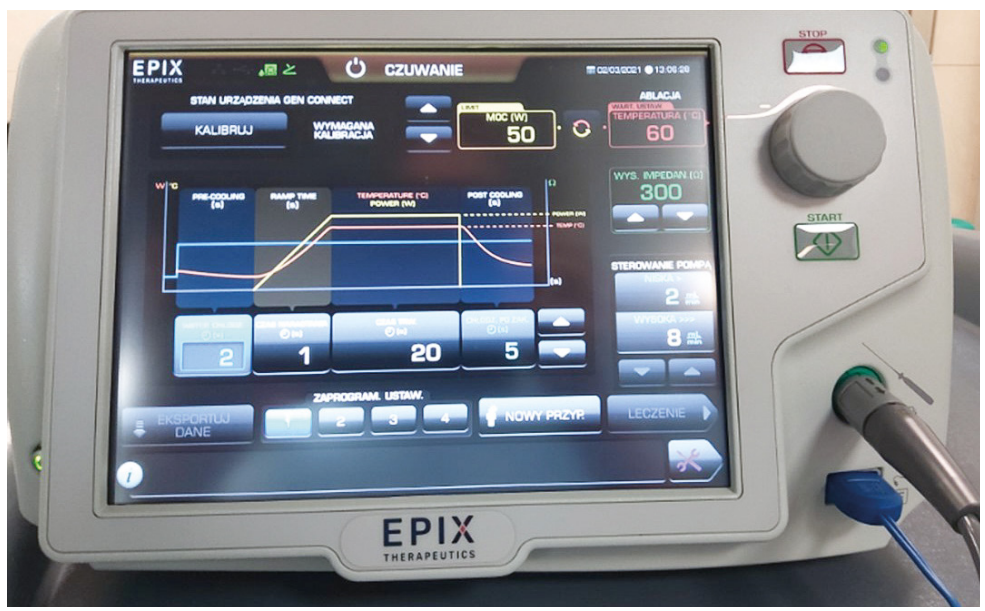

Figure 3. DiamondTemp RF generator. 
Specifically, we described the practical use of a new, diamond-tipped catheter which allows for high-power short-duration ablation, where the amount of energy delivered depends on the temperature in the tissue adjacent to the catheter tip. Therefore, this method can also be defined as delivering the right power for the right duration. Our aim was to present a new technology related to the DiamontTemp catheter and its first use in Poland in patients undergoing pulmonary vein isolation.

\section{Materials and methods}

First we performed wet-lab tests with DiamondTemp catheter (Fig. 4-5) to visualize lesion formation and to evaluate the risk of steam-pop during high-power ablation. The next day we performed our first 3 clinical procedures (Fig. 6-10).

The first patient was a 46-yearold male with 4-year history of lone long-term persistent AF. The second patient was 26-year-old male with 2-year history of paroxysmal AF and previous ablation of the slow pathway, because of paroxysmal atrioventricular nodal reentrant tachycardia. The third patient was 61-year-old male with arterial hypertension, hyperlipidemia and coronary artery disease (NSTEMI treated with $\mathrm{PCl} 7$ years ago) and paroxysmal AF diagnosed 7 years ago. All 3 patients were highly symptomatic (EHRA score 3). In the first 2 patients, typical transseptal punctures were performed followed by left atrial mapping with the Advisor catheter (Abbott) using EnSite-Precision system (Abbott) (Fig. 6). The third patient had a residual atrial septal leak, therefore we attempted to ablate without fluoroscopy (Fig. 7). Anatomical mapping of the right atrium was performed. Based on the images obtained, both catheters (the DiamondTemp and Advisor) were introduced into the left atrium through the leak in the septum using technique presented in previous publication [17]. In all 3 patients pulmonary vein isolation was performed us-

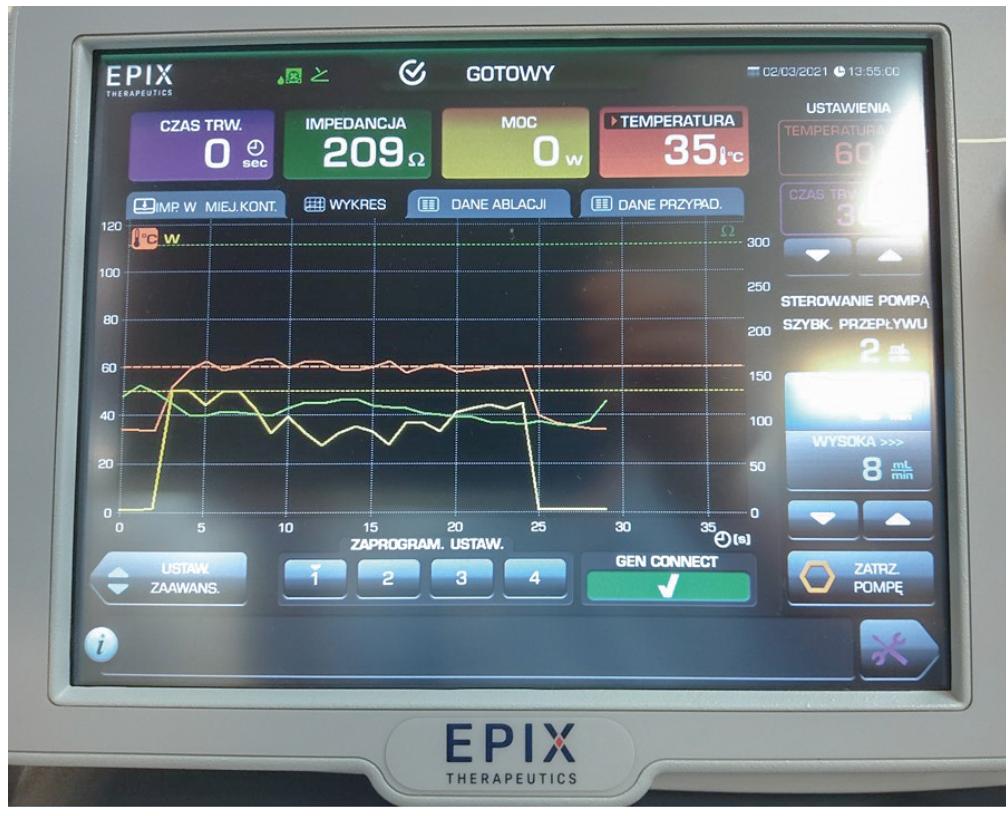

Figure 4. Screen presenting the entire progress of RF application in wet lab. The first two seconds show the saline infusion ( $8 \mathrm{~mm} / \mathrm{min}$ ), after which the RF application started. $50 \mathrm{~W}$ were reached within $1 \mathrm{~s}$. After 2 seconds of $50 \mathrm{~W}$ application, the maximal temperature of $60^{\circ} \mathrm{C}$ was reached and the power was then reduced. From that point, the power was kept at an optimal level to stabilize the temperature at $60^{\circ} \mathrm{C}$. In the last second the power was reduced to $0 \mathrm{~W}$. From 25 seconds shown on the diagram, 23 seconds depict the achievement of optimal power. Red line - temperature, yellow line - power, green line - impedance.

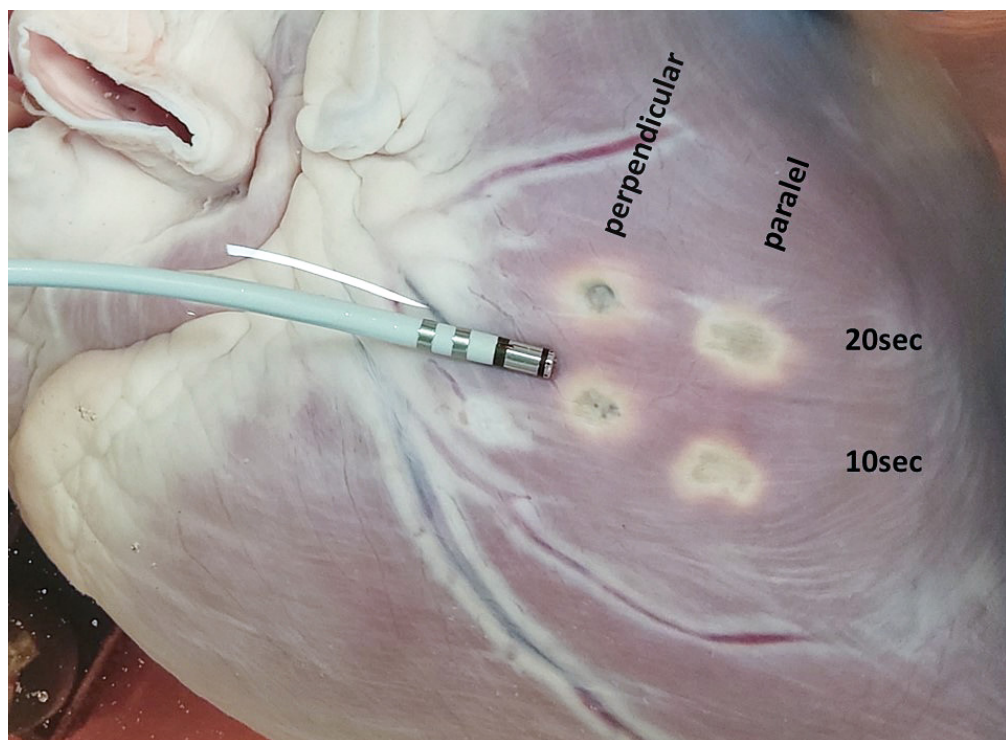

Figure 5. Final results of four applications of DiamondTemp RF, performed with different settings. The photo presents the surface area of a pig's heart post-application.

ing the "close protocol" (the distance between neighboring ablation points was $<6 \mathrm{~mm}$ ) [18] using DiamondTemp catheter (Medtronic) with nominal high-power $(50 \mathrm{~W})$ under temperature control (Fig. 8-10). 


\section{Results}

The procedure (and fluoroscopy) duration were, respectively: 146.0 (8.9), 177.0 (5.9), $132.0(0.0) \mathrm{min}$. The dose area product (DAP) were: $218.5,68.1$ and $0.0 \mathrm{mGy}$. To further illustrate the presented values, we compare them to the parameters recorded in the reference group of 10 patients which underwent point-by-point similar procedures using the classical technique. The values for this group were respectively: 143.0 $\pm 27.0 \mathrm{~min}(6.0 \pm 4.4 \mathrm{~min})$, DAP $82.1 \pm 54.4 \mathrm{mGy}$.

The number of RF application was 73, 203 and 117 (in the reference group it was $122 \pm 20$ ), duration application was 14.7, 32.7, $30.8 \mathrm{~min}$ (reference group $37.3 \pm 11.4$ ). Power average (range) was: 50 (49-53), 49 (30-55), 50 (45-55) W, temperature 47 (45-52), 48 (40-60), $45(40-60)^{\circ} \mathrm{C}$.

Procedural endpoints were achieved in all but one patient with incomplete isolation of the low segment of the right inferior pulmonary vein. In this patient the "close protocol" was ended, and few accessory applications were performed in lower region of this vein, but with no effect. During pacing from the pulmonary vein a new map was obtained and the earliest atrial activation was confirmed at lower part of the right inferior pulmonary vein ostium. Serial application in this region did not terminate atrial capture during pacing from the vein. There were no procedural complications noted.

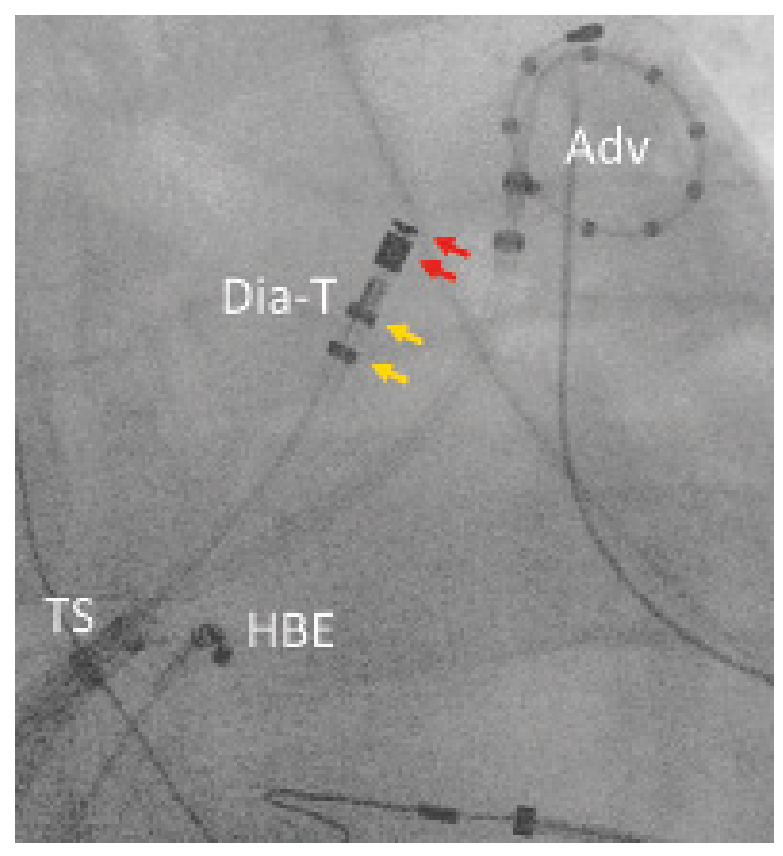

Figure 6. X-ray from the first clinical procedure using DiamondTemp ablation system. Adv - Advisor circular multipolar mapping catheter in the left superior pulmonary vein. Dia-T - the DiamondTemp catheter in the left atrial roof proximal to the left superior pulmonary vein ostium. Red arrows - two distal RF electrodes, yellow arrows - proximal diagnostic ring electrodes, HBE - the quadripolar catheter in His bundle position, TS - transseptal sheath.

\section{Discussion}

The DiamondTemp catheter is a new generation $7.5 \mathrm{~F}$ saline-irrigated catheter with a real time power modulation in the temperature control mode. Distal RF electrode tip is $4.1 \mathrm{~mm}$ and comprised of platinum/iridium $(90 \% / 10 \%)$ and chemical vapor deposition diamond network. This tip has two components separated by a $0.5 \mathrm{~mm}$ ring of chemical vapor deposition diamond. The distal electrode segment is $0.6 \mathrm{~mm}$ and contains 3 thermally insulated external thermocouples and 6 saline irrigation ports. Proximal electrode segment is $3.0 \mathrm{~mm}$ and also contains 3 thermocouples spaced equally around the proximal edge of the RF electrode and ring of chemical vapor deposition diamond at the proximal edge of the RF electrode for additional cooling. Heat and cooling
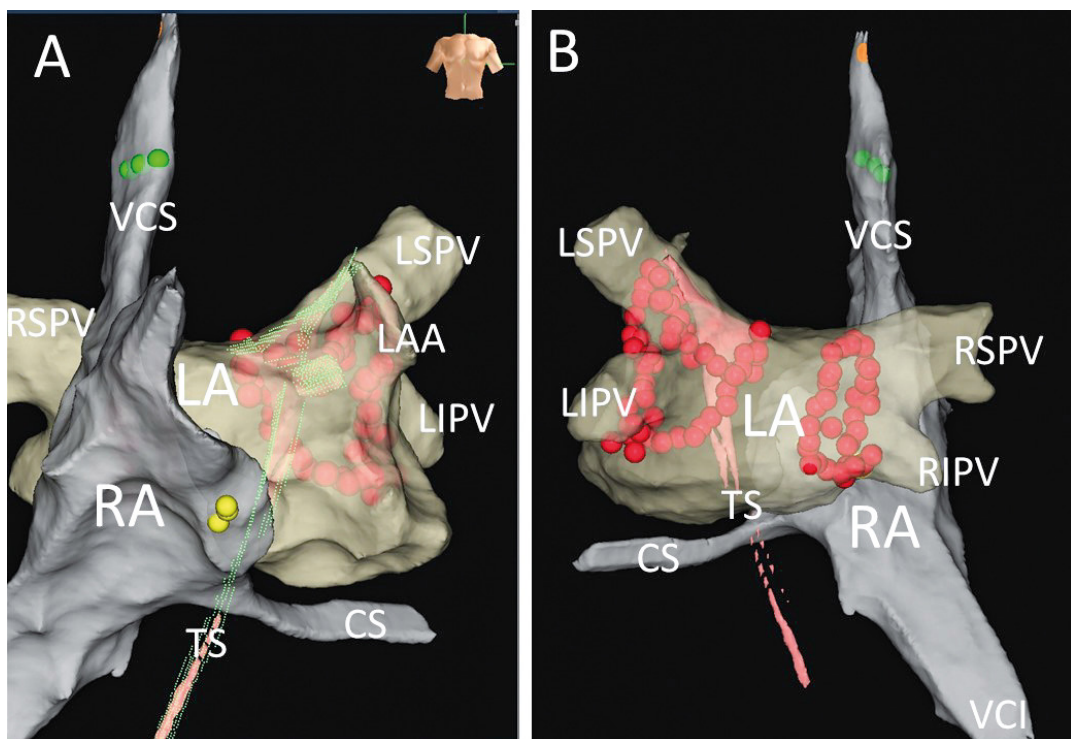

Figure 7. Final results presented with EnSite system's anatomical maps, exhibiting lesions resulting from pulmonary vein isolation, performed with no fluoroscopy using DiamondTemp catheter. Panel A - AP view, panel B - PA view. LA - the left atrial map, RA - the right atrial map, TS - map presenting the path of catheter through the PFO. CS - the coronary sinus, LAA - the left atrial appandage, LIPV - the left inferior pulmonary vein, LSPV - the left superior pulmonary vein, RIPV - the right inferior pulmonary vein, RSPV - the right superior pulmonary vein, $\mathrm{VCl}$ - the inferior vena cava, $\mathrm{VCS}$ - the superior vena cava. Yellow dots - His bundle position, red dots - ablation points. Both lines performed with the „close protocol” (the distance between points $<6 \mathrm{~mm}$ ). Green points - points of successful pacing of the phrenic nerve. 
transfer are 200-400 times faster with diamond than with platinum-iridium. Extremely high thermal diffusivity allows to quick conduction of thermal energy through the diamond shunt network. Little to no heat is retained at the catheter tip to be cooled, therefore allowing a lower irrigation flow rate $(8 \mathrm{ml} / \mathrm{min}$ ) for all power delivery. This combined effect of rapid catheter tip cooling driven by the diamond shunt network and a low-flow irrigation rate allows for safe, effective and efficient lesion formation.

Temperature from each of the 6 thermocouples is sampled every 20 msec (50 times per second) and RF generator modulates power delivery based on the highest sensor temperature of all thermocouples. RF generator delivers $50 \mathrm{~W}$ to achieve set-point temperature (optimal value is $60^{\circ} \mathrm{C}$ ). When temperature set-point is achieved the generator modulates power to maintain set temperature.

Because temperature provides direct feedback about lesion formation [10-12], in this model we do not need catheter-tissue pressure control. If the pressure is higher and temperature is going to reach set-point, the power was reduced (Fig. 9). Whereas when the contact was poor, we did not observe enough temperature rise (Fig. 8). In high power ablation the best method for the assessment of lesion efficacy is the change in electrogram (ECG) voltage. The duration of the application should be about 3.0-5.0 sec longer than potential disappearances or is significantly reduced (at least 75$80 \%$ ) [8, 19] (Fig. 10). To reduce the influence of far-field potentials on the local signal amplitude, the high-resolution ECG is crucial. For this purpose, the distal RF electrode was divided into two segments, as described above. If the reduction of the local potential is inconclusive (e.g. during atrial fibrillation, RF current artifacts), the evaluation of the effectiveness of the application can be carried out based on changes in impedance [20].

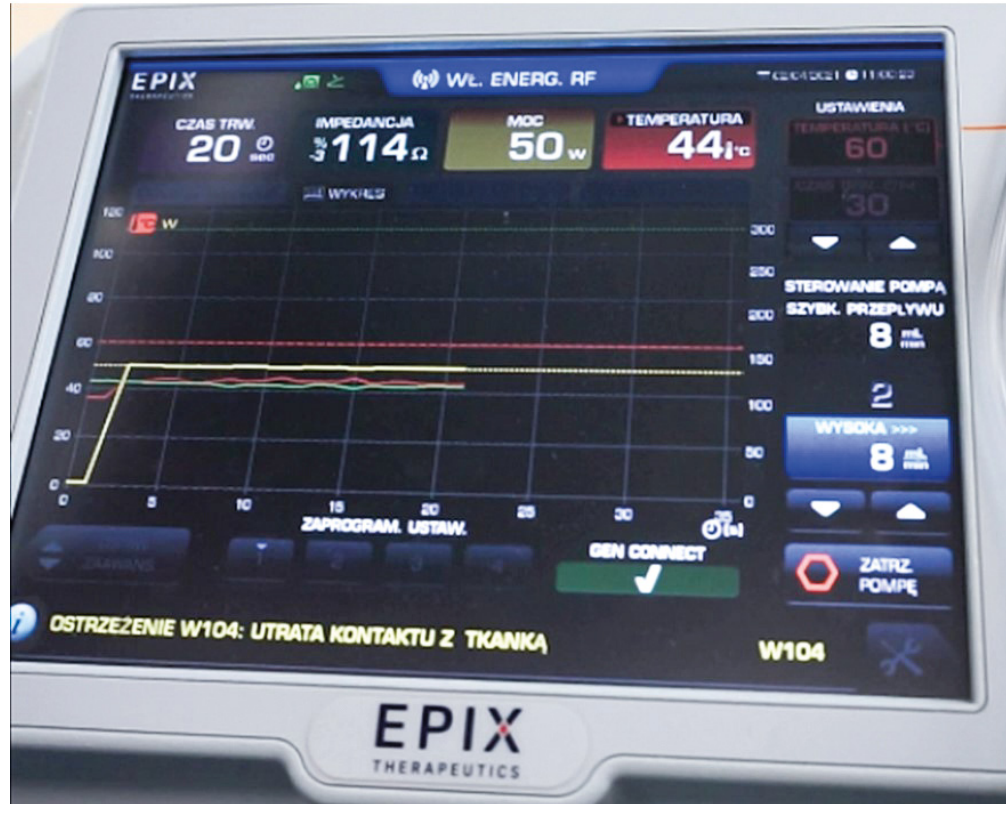

Fig.8. RF power generator monitor during one of the applications. The last second of a 20 second RF application presents insufficient pressure of the catheter against cardiac tissue. A relatively low temperature despite the constant $50 \mathrm{~W}$ and the lack of a sufficient decrease in impedance suggests ineffective application. Time of application: 20 sec, Impedance:114 ohms (decrease of $3 \mathrm{ohms}$ compared to the start of application), Power: $50 \mathrm{~W}$, Temperature: $44^{\circ} \mathrm{C}$. Yellow line - power, red line - temperature, green line - impedance. Saline infusion via DiamondTemp catheter - $8 \mathrm{ml} / \mathrm{min}$. Marker W104 - system suggests loss of contact between the catheter and tissue.

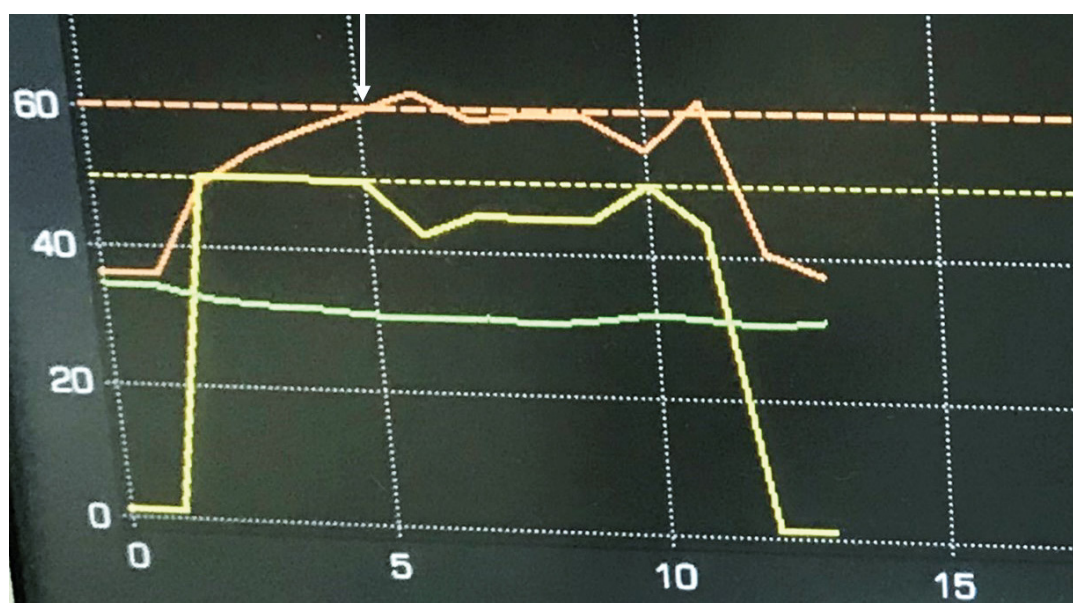

Fig.9. DiamondTemp RF Generator monitor after a successful application is completed. Power of 50W, Temperature achieved: $60^{\circ} \mathrm{C}$ after $5 \mathrm{sec}$. When temperature set-point is achieved (the white arrow) the generator modulated power to maintain set temperature (in this case between 41 and 50W). Due to optimal tissue contact and early dissappearence of local signal, the application was terminated after 10 seconds. Yellow line - power, red line - temperature, green line - impedance.

Significant tissue heating is associated with a predictable fall in impedance. Impedance drop of 5-10\% can offer an independent means of assessing the true outcome [20]. 

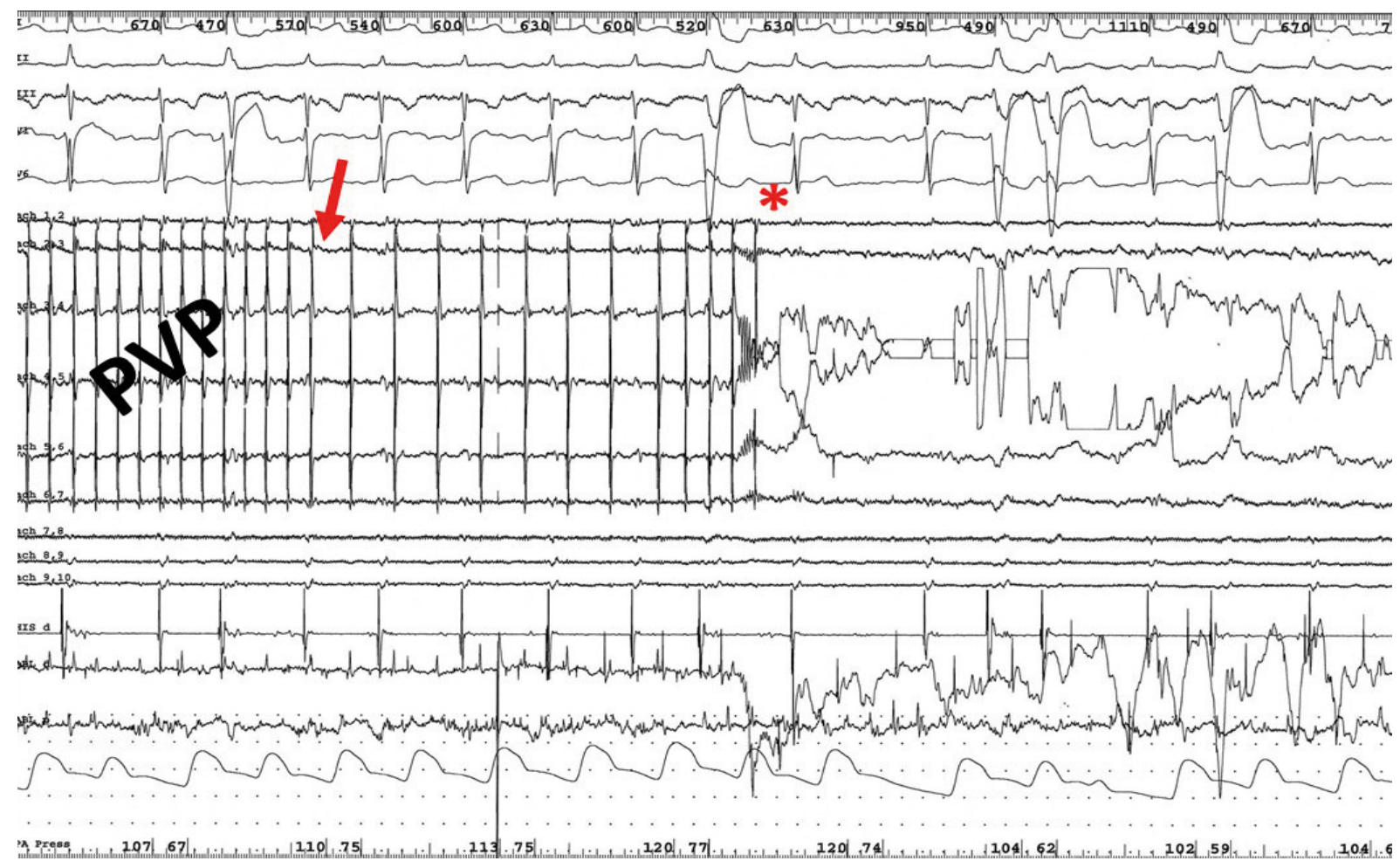

Fig.10. Disappearance of the pulmonary vein potentials during RF current application from DiamondTemp ablation system, preceded by their gradual slowing down in the first patient diagnosed with persistent atrial fibrillation. Arrow - the beginning of the pulmonary vein potentials slow down, star - disappearance of the pulmonary vein potentials. PVP - pulmonary vein potentials

In the TRAC-AF study [8] on porcine model, the investigators presented lesion transmurality created by the DiamondTemp catheter in 51 of 55 lesions $(92,7 \%)$ performed in 6 pigs. In the second part of the study, the DiamondTemp catheter was used in 35 patients at a single center. The ablation was performed in temperature-control mode $\left(60^{\circ} \mathrm{C} / 50\right.$ $W$ ) with the goal of achieving $80 \%$ reduction in high-resolution electrogram (EGM) amplitude. All pulmonary veins were successfully isolated during the procedure. Compared with the retrospective control group (standard force-sensing ablation catheter), the study cohort had significantly shorter mean RF application duration (average difference $33.0 \mathrm{~min}$ ), fluoroscopy time and lower acute dormant pulmonary veins reconduction. At 3 months, 23 patients underwent remapping. In 17 of 23 patients (73.9\%) and 39 of 46 pulmonary veins pairs (84.8\%) remained durably isolated.

The largest clinical evaluation of the DiamondTemp catheter was DIAMOND-AF study [9]. It was FDA-regulated, prospective, multicenter $(n=23)$, single-blind, noninferiority, randomized (1:1) controlled trial designed to compare the safety and effectiveness of the DiamondTemp ablation system with a contact force sensing ablation system in patients with drug-refractory symptomatic paroxysmal AF. A total of 482 patients were randomized (239 DiamondTemp catheter ablation, 243 control). Acute PVI was confirmed in 239 subjects $(100 \%)$ in the DiamondTemp ablation group and 241 subjects (99.2\%) in the control group. Total procedure, fluoroscopy, and left atrial dwell duration were similar between arms. Total RF time and individual RF ablation duration were significantly shorter in the DiamondTemp group and significantly less fluid was infused through the DiamondTemp catheter. Complications occurred in $8(3.3 \%)$ patients treated with DiamondTemp catheter and 16 (6.6\%) patients in control group. In the DiamondTemp group there were 2 cardiac tamponades, 2 TIA episodes, 1 permanent phrenic nerve paralysis, 1 vagal nerve injury and 2 vascular complications. In control group there were 2 cardiac tamponades, 1 pericarditis, 1 pulmonary edema, 1 stroke, 1 TIA and 4 vascular complications. There was no deaths. After 1 year follow up (with 3-month blanking period) free of atrial arrhythmias lasting $\geq 30.0 \mathrm{sec}$ were 189 (79.1\%) patients after ablation with the DiamondTemp catheter and 184 (75.7\%) patients in the control group.

\section{Conclusions}

The DiamondTemp saline-irrigated catheter seems to be safe and effective for high-power short-duration ablation in patients with atrial fibrillation. This must be confirmed in a larger group of patients. This technique makes it possible to complete the procedure with zero fluoroscopy exposure. 


\section{References}

1. Leshem E, Zilberman I, Tschabrunn CM, Barkagan M, Contreras-Valdes FM, Govari A, et al. High-Power and Short-Duration Ablation for Pulmonary Vein Isolation. JACC Clin Electrophysiol [Internet]. 2018 Apr;4(4):467-79. Available from: https://www.jacc.org/doi/full/10.1016/i.jacep.2017.11.018

2. Barkagan $\mathrm{M}$, Contreras-Valdes $\mathrm{FM}$, Leshem $\mathrm{E}$, Buxton $\mathrm{AE}$, Nakagawa $\mathrm{H}$, Anter E. High-power and short-duration ablation for pulmonary vein isolation: Safety, efficacy, and long-term durability. J Cardiovasc Electrophysiol [Internet]. 2018 Sep 20;29(9):1287-96. Available from: https://onlinelibrary.wiley.com/doi/abs/10.1111/ice.13651

3. Vassallo F, Meigre LL, Serpa E, Cunha C, Simoes A, Carloni H, et al. Changes and impacts in early recurrences after atrial fibrillation ablation in contact force era: comparison of high-power short-duration with conventional technique-FIRST experience data. J Interv Card Electrophysiol [Internet]. 2020 Nov 5; Available from: http://link.springer.com/10.1007/ s10840-020-00911-x

4. Dikdan SJ, Junarta J, Bodempudi S, Upadhyay N, Pang Z, Frisch DR. Comparison of clinical and procedural outcomes between high-power short-duration, standard-power standard-duration, and temperature-controlled noncontact force guided ablation for atrial fibrillation. J Cardiovasc Electrophysiol [Internet]. 2021 Mar 19;32(3):608-15. Available from: https://onlinelibrary.wiley.com/doi/10.1111/ice.14868

5. Kewcharoen J, Techorueangwiwat C, Kanitsoraphan C, Leesutipornchai T, Akoum N, Bunch TJ, et al. High-power short duration and low-power long duration in atrial fibrillation ablation: A meta-analysis. J Cardiovasc Electrophysiol [Internet]. 2021 Jan 18;32(1):71-82. Available from: https://onlinelibrary.wiley.com/doi/10.1111/ice.14806

6. Chen C, Wu J, Jin C, Liu M, Xu Y. Comparison of high-power short-duration and low-power long-duration radiofrequency ablation for treating atrial fibrillation: Systematic review and meta-analysis. Clin Cardiol [Internet]. 2020 Dec 27;43(12):1631-40. Available from: https://onlinelibrary.wiley.com/doi/10.1002/clc.23493

7. Reddy VY, Grimaldi M, De Potter T, Vijgen JM, Bulava A, Duytschaever MF, et al. Pulmonary Vein Isolation With Very High Power, Short Duration, Temperature-Controlled Lesions. JACC Clin Electrophysiol [Internet]. 2019 Jul;5(7):778-86. Available from: https://linkinghub.elsevier.com/retrieve/pii/S2405500X19303032

8. Iwasawa J, Koruth JS, Petru J, Dujka L, Kralovec S, Mzourkova K, et al. Temperature-Controlled Radiofrequency Ablation for Pulmonary Vein Isolation in Patients With Atrial Fibrillation. J Am Coll Cardiol [Internet]. 2017 Aug;70(5):542-53. Available from: https://linkinghub.elsevier.com/retrieve/pii/S0735109717377331

9. Kautzner J, Albenque J-P, Natale A, Maddox W, Cuoco F, Neuzil P, et al. A Novel Temperature-Controlled Radiofrequency Catheter Ablation System Used to Treat Patients With Paroxysmal Atrial Fibrillation. JACC Clin Electrophysiol [Internet]. 2021 Mar;7(3):352-63. Available from: https://linkinghub.elsevier.com/retrieve/pii/S2405500X20311907

10. Nath S, Dimarco JP, Haines DE. Basic Aspects of Radiofrequency Catheter Ablation. J Cardiovasc Electrophysiol [Internet]. 1994 Oct;5(10):863-76. Available from: http://doi.wiley.com/10.1111/i.1540-8167.1994.tb01125.x

11. Haines DE. The Biophysics of Radiofrequency Catheter Ablation in the Heart: The Importance of Temperature Monitoring. Pacing Clin Electrophysiol [Internet]. 1993 Mar;16(3):586-91. Available from: http://doi.wiley. com/10.1111/j.1540-8159.1993.tb01630.x

12. Hindricks G, Haverkamp W, Gulker H, Rissel U, Budde T, Richter KD, et al. Radiofrequency coagulation of ventricular myocardium: Improved prediction of lesion size by monitoring catheter tip temperature. Eur Heart J [Internet]. 1989 Nov;10(11):972-84. Available from: https://academic.oup.com/eurheartj/article/411148/Radiofrequency

13. Walczak F, Szufladowicz E, Jedynak Z, Kępski R, Koźluk E, Łastowiecka E, et al. Ablacja prądem wysokiej częstotliwości drogi o wolnym przewodzeniu u chorych z nawrotnym częstoskurczem węzłowym - doniesienie wstępne [in Polish]. Kardiol Pol. 1993;38:199-204.

14. Kozluk E, Walczak F, Szufladowicz E, Kozluk J, Jedynak Z, Kepski R. Time of radiofrequency current application necessary for permanent damage of the slow conducting pathway. Kardiol Pol. 1994;41:117-8.

15. Nakagawa H, Yamanashi WS, Pitha J V., Arruda M, Wang X, Ohtomo K, et al. Comparison of In Vivo Tissue Temperature Profile and Lesion Geometry for Radiofrequency Ablation With a Saline-Irrigated Electrode Versus Temperature Control in a Canine Thigh Muscle Preparation. Circulation [Internet]. 1995 Apr 15;91(8):2264-73. Available from: https://www. ahajournals.org/doi/10.1161/01.CIR.91.8.2264

16. Koźluk E, Piątkowska A, Rodkiewicz D, Opolski G. High-power and short-duration ablation with QDOT Micro QMODE+ algorithm for pulmonary vein isolation and the right superior ganglion plexus ablation with zero-fluoroscopy. Eur J Transl Clin Med. 2021;4(2). Available from: https://ejtcm.gumed.edu.pl/articles/94

17. Koźluk E, Łojewska K, Hiczkiewicz J. First experience with left atrial arrhythmia ablation using a bidirectional steerable transseptal sheath (Vizigo) visible in the CARTO system as a method to reduce fluoroscopy. Eur J Transl Clin Med [Internet]. 2020;3(2):18-21. Available from: http://dx.doi.org/10.31373/ejtcm/131049 
18. Phlips T, Taghji P, El Haddad M, Wolf M, Knecht S, Vandekerckhove $\mathrm{Y}$, et al. Improving procedural and one-year outcome after contact force-guided pulmonary vein isolation: the role of interlesion distance, ablation index, and contact force variability in the 'CLOSE'-protocol. EP Eur [Internet]. 2018 Nov 1;20(FI_3):f419-27. Available from: https://academic.oup. com/europace/article/20/FI 3/f419/4791151

19. Havranek S, Alfredova H, Fingrova Z, Souckova L, Wichterle D. Early and Delayed Alteration of Atrial Electrograms Around Single Radiofrequency Ablation Lesion. Front Cardiovasc Med [Internet]. 2019 Jan 8;5. Available from: https://www. frontiersin.org/article/10.3389/fcvm.2018.00190/full

20. Strickberger SA, Ravi S, Daoud E, Niebauer M, Man KC, Morady F. Relation between impedance and temperature during radiofrequency ablation of accessory pathways. Am Heart J [Internet]. 1995 Nov;130(5):1026-30. Available from: https://linkinghub.elsevier.com/retrieve/pii/000287039590204X 